\title{
30 Jahre dermatoonkologische Virologie
}

H. zur Hausen
Papillomviren als Krebserreger

30 Years of Dermatooncological Virology - Papillomavirus as Cause of Cancer

\section{Zusammenfassung}

In den vergangenen 30 Jahren wurden bestimmte Typen der sehr heterogenen humanen Papillomvirus (HPV) Familie mit spezifischen Krebserkrankungen des Menschen in Zusammenhang gebracht und inzwischen für einige weit verbreitete Krebsformen als auslösende Ursache identifiziert. Hier handelt es sich vorrangig um den Gebärmutterhalskrebs, aber auch um weitere anogenitale Krebserkrankungen, sowie um etwa ein Viertel aller Mundhöhlenkarzinome. In zunehmendem Maße zeichnet sich auch eine Beteiligung bestimmter Papillomvirus-Typen für die Auslösung von Plattenepithelkarzinomen der Haut ab. Diese Typen sind in der Lage, die durch UV-Licht bedingte ausgelöste Apoptose (den programmierten Tod der geschädigten Zelle) zu unterdrücken und damit weiteres Zellwachstum mutierter Zellen zu ermöglichen. Die so genannten „Hochrisikotypen“ dieser Virusfamilie, mit den Prototypen HPV 16 und 18, erweisen sich als wirksame Karzinogene, auch wenn nur ein geringer Prozentsatz der infizierten Frauen später an Gebärmutterhalskrebs erkrankt. In der großen Mehrzahl infizierter Personen wird die Infektion über Immunmechanismen erfolgreich kontrolliert. Diese Viren besitzen eigene Krebsgene (Onkogene), deren Wirkungsmechanismus heute zunehmend besser verstanden wird. Global gesehen lassen sich etwa 13 -15\% aller bei Frauen auftretenden Krebserkrankungen ursächlich auf Papillomvirus-Infektionen zurückführen. Inzwischen wurden wirksame vorbeugende Impfstoffe gegen die bedeutsamsten pathogenen Vertreter dieser Virusfamilie entwickelt, die in den kommenden zwei Jahren allgemein verfügbar werden dürften. Deren breite Anwendung lässt auf eine wirksame Kontrolle des Gebärmutterhalskrebses und seiner Vorstufen hoffen. Bisherige Versuche, auch therapeutisch effiziente Impfstoffe zu entwickeln, erwiesen sich bisher als weniger erfolgreich.

\section{Abstract}

During the past 30 years specific types of human papillomaviruses (HPV) have been linked to certain human cancers and have been identified as causative agents of these malignant proliferations. Primarly cancer of the cervix, but also other anogenital cancers and approximately $25 \%$ of oral cancers are etiologically related to these infections. Increasing evidence points to an indirect role of other HPV infections in squamous cell carcinomas of the skin. Some of the latter types suppress apoptosis, caused by DNA damage due to UV exposure of the skin, and enable continued proliferation of mutated cells. Anogenital high risk types like HPV16 and 18 are effective carcinogens, although only a small percentage of infected women eventually will develop cancer of the cervix. Immunological surveillance appears to result in clearance of most of these infections. These viruses express their own oncogenes. The mechanism of their oncogenic function is presently partially understood. Globally about $13-15 \%$ of all cancers in women are caused by HPV infections. Effective preventive vaccinations against the most prevalent high risk HPV types are presently in clinical trials. It is anticipated that they will become available in 2006 or 2007. It is expected that they will lead to an effective control of cervical cancer and its precursor lesions, if vaccination is applied to juveniles before onset of sexual activity. Present approaches for therapeutic vaccinations have been less successful and require further studies. 
Obwohl bereits in den 30er-Jahren des vergangenen Jahrhunderts die Krebsauslösung durch Papillomviren bei amerikanischen Wildkaninchen beschrieben und intensiv analysiert wurde, vergingen weitere 45 bis 50 Jahre, bevor die intensive Untersuchung über eine mögliche Beteiligung dieser Virusgruppe bei Krebserkrankungen des Menschen einen dann eine eher stürmische Entwicklung nahm [1]. Auslösende Ursache war die Identifizierung neuer Typen dieser Virusfamilie aus Biopsien des Gebärmutterhalskrebses [2,3].

In der Folge hat sich diese Familie als außerordentlich heterogen erwiesen, zurzeit ist die DNA von 106 humanen Papillomvirus(HPV)-Typen in ihrer Basenfolge analysiert [4] (und de Villiers, pers. Mitteilung), wobei zu erwarten ist, dass diese Zahl sich in Zukunft noch deutlich erhöhen wird, da bereits jetzt von einer Reihe von weiteren Typen die Partialsequenz vorliegt. Konsistente Nachweise von spezifischen Papillomvirus-Infektionen ließen sich bis jetzt für Krebserkrankungen des Gebärmutterhalses, des Anus, für bestimmte histologische Formen des Vulva- und Peniskrebses und der Scheide führen. Die gleichen Typen werden auch in 20-25\% von Mundhöhlenkrebserkrankungen (einschließlich Tonsillen) und beim sehr seltenen Nagelbettkrebs nachgewiesen. Nach gegenwärtigem Kenntnisstand können wir davon ausgehen, dass zwischen Papillomvirusinfektion und diesen Krebserkrankungen ein kausaler Zusammenhang besteht [5].

Ein breites Interesse finden derzeit Befunde, die auf ein interessantes Zusammenwirken weiterer Papillomvirus-Typen mit physikalischen krebsauslösenden Faktoren, dem ultravioletten Anteil des Sonnenlichtes, bei der Entstehung von Plattenepithelkarzinomen der Haut deuten lassen. Die Anschaltung zellulärer Reparatursystem nach Schädigung der Haut durch intensive Sonneneinstrahlung aktiviert gleichzeitig latent vorhandene Papillomvirustypen, wobei ein aktiviertes virales Genprodukt, das sog. E6 Protein, durch Bindung an proapoptotische mitochondriale Proteine deren Funktion unterdrückt und damit die weitere Vermehrung vorgeschädigter Zellen ermöglicht.

Weniger gut belegt sind dagegen sporadische Berichte über Papillomvirus-positive Karzinome des Dickdarms, der Brust, der Lunge, Blase, Prostata, des Ovars, der Nasennebenhöhlen, des Larynx und der Speiseröhre. Obwohl für einzelne dieser Organsysteme durchaus wohl dokumentierte Befunde vorliegen, erlauben die vorliegenden Ergebnisse, einschließlich einer Reihe von Negativberichten für Karzinome der betreffenden Organe, keine abschließende Bewertung.

\section{Anogenitale Papillomvirus-Infektionen}

Experimentell ergaben sich frühzeitig Anhaltspunkte für eine Rolle von Papillomviren beim Gebärmutterhalskrebs. Neben dem konsistenten Nachweis von viraler DNA in Krebsbiopsien und Gewebekultur-Zelllinien von Zervixkarzinomen, vor allem der HPV Typen 16 und 18, ließen sich auch regelmäßig Transkripte dieser Viren in den betroffenen Materialien nachweisen $[3,6]$. Abb. 1 zeigt die Genomstruktur von Papillomviren mit den Onko-

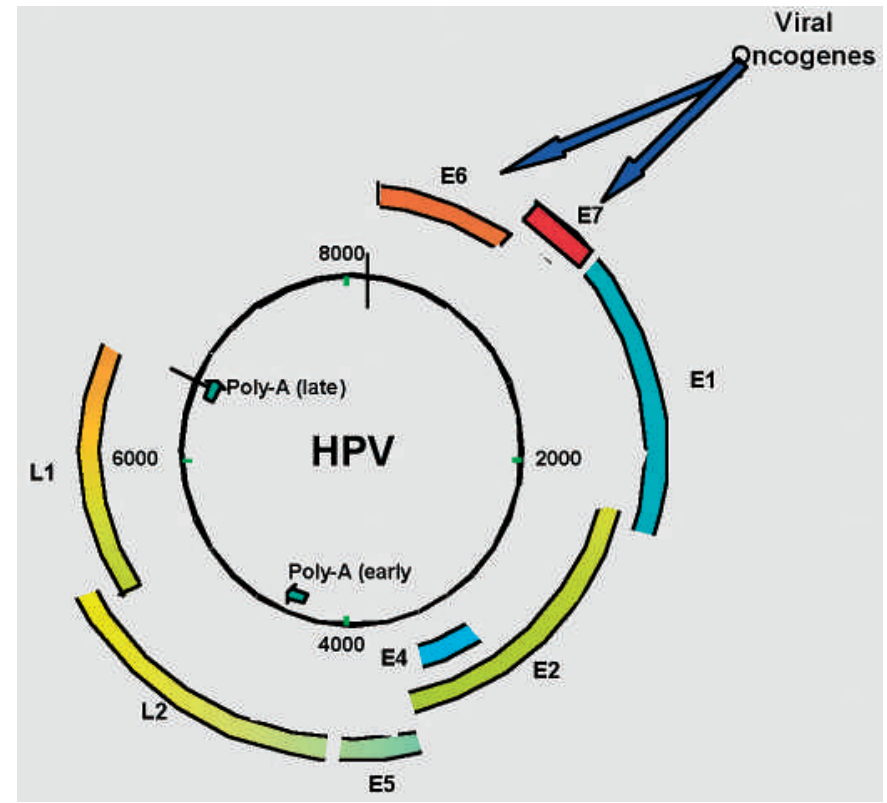

Abb. 1 Schematische Darstellung der Organisation von HPV-Genomen. Die farbigen Bereiche stellen offene Leseraster dar, die für Proteine kodieren.

genen E6 und E7, die regelmäßig in Gebärmutterhalskarzinomen aktiv sind.

Die gleichen Gene sind in der Lage, normale menschliche Zelle zu „immortalisieren“, d.h., sie in der Gewebekultur unbegrenzt wachsen zu lassen [7]. Schließlich lässt sich zeigen, dass ihre Ausschaltung durch interferierende RNA, durch Injektion spezifischer Antikörper oder durch Protein-Aptamere zum Zelltod oder in die Seneszenz führt, das maligne Wachstum also unterbrochen wird.

Epidemiologisch sind es vor allen drei Hinweise, die die Rolle dieser Viren unterstützen: Typ-spezifische Fall-/Kontroll-Studien, prospektive Kohorten Studien und schließlich die phylogenetische Verwandtschaft. Unter Berücksichtigung der hier vorliegenden Ergebnisse lassen sich die Typen 16, 31, 33, 35, 39, 45, 51, 52, 56, 58, 59 und 66 als Hochrisiko-Typen ausweisen, deren kausale Rolle an der Auslösung des Zervixkarzinoms nicht mehr bestritten wird. Typ 16 dominiert bei weitem und dürfte global gesehen für annähernd $53 \%$ der Fälle verantwortlich sein. Typ 18 folgt mit $17 \%$, Typ 31 mit etwa 7, Typ 33 und 52 mit annähernd $3 \%$, Typ 58 mit $2 \%$, während alle anderen nur eine kleine Fraktion ausmachen [8]. Vermutlich gehören auch noch die Typen 26, 68, 73 und 82 in diese Gruppe, die vorliegenden Ergebnisse erlauben jedoch gegenwärtig nicht deren Zuordnung.

Vor allem die Typen 16 und 18 spielen auch bei anderen Krebserkrankungen des Anogenitalbereichs, der Mundhöhle und der Haut eine Rolle. Tab. 1 gibt eine entsprechende Übersicht.

Es darf an dieser Stelle nicht vergessen werde, dass Viren, die genitale Warzen (Condylomata acuminata) und Larynxpapillomatosis auslösen können, HPV 6 und 11, an den seltenen invasiv wachsenden Buschke-Löwenstein Tumoren des Anogenitalbereichs und an verrukösen Karzinomen des Larynx und der Bron- 
Tab. 1 Beteiligung anogenitaler Papillomvirus-Typen an Krebserkrankungen des Menschen. Die fett gedruckten Zahlen weisen auf prävalente Typen bei den entsprechenden Erkrankungen.

\begin{tabular}{|c|c|c|}
\hline Krebserkrankung & $\begin{array}{l}\text { Beteiligte } \\
\text { Papillomvirus-Typen }\end{array}$ & Prozent HPV-positiv \\
\hline Zervixkarzinom & $\begin{array}{l}\mathbf{1 6}, \mathbf{1 8}, 31,33,35,39 \\
45,51,52,56,58,59 \\
66(26,68,73,82)\end{array}$ & $>95 \%$ \\
\hline $\begin{array}{l}\text { Vulva-Karzinom } \\
\text { Basaloid } \\
\text { „Warty“ } \\
\text { keratinisierend }\end{array}$ & $\begin{array}{l}16,18 \\
16,18 \\
16\end{array}$ & $\begin{array}{l}>50 \% \\
>50 \% \\
<10 \%\end{array}$ \\
\hline $\begin{array}{l}\text { Penis-Karzinom } \\
\text { Basaloid } \\
\text { „Warty“ } \\
\text { keratinisierend }\end{array}$ & $\begin{array}{l}\mathbf{1 6}, 18 \\
\mathbf{1 6}, 18 \\
\mathbf{1 6}\end{array}$ & $\begin{array}{l}>50 \% \\
>50 \% \\
<10 \%\end{array}$ \\
\hline Vagina & 16,18 & $>50 \%$ \\
\hline Anus & 16,18 & $>70 \%$ \\
\hline Mundhöhle und Tonsillen & $16,18,33$ & $\sim 25 \%$ \\
\hline Nagelbett & 16 & $\sim 70 \%$ \\
\hline
\end{tabular}

chien nach häufig rezidivierender Larynxpapillomatose beteiligt sein können.

Der Mechanismus, über den Hochrisiko-Papillomviren (Hr-HPV) Krebs verursachen können, wird zunehmend besser verstanden, auch wenn die Ursachen für Viruspersistenz nicht völlig abgeklärt sind. Etwa $10 \%$ infizierter Frauen weisen über zwei Jahre persistiernde Infektionen auf, die in der Folge in zervikale intraepitheliale Neoplasien (CIN) übergehen können $[9,10]$. Hr-HPV Infektionen führen zu hochgradigen Neoplasien (CIN III) mit hohem Risiko der weiteren malignen Entartung. CIN I und CIN II sind häufig auch durch andere Papillomvirus-Typen bedingt. In diesen Fällen kommt es besonders oft zu spontaner Rückbildung.

Im Verlauf der Entwicklung von CIN III Läsionen und beim späteren Übergang in invasiv wachsende Tumoren findet in gut 70\% die Integration der viralen DNA in das Wirtszellgenom statt. Der Integrationsvorgang fördert die Synthese viraler Onkoproteine ohne direkt für malignes Wachstum verantwortlich zu sein. Wenn die Karzinomentstehung ohne virale Integration zustande kommt, werden ebenfalls die viralen Onkogene E6 und E7 vermehrt synthetisiert, offensichtlich über den Verlust zellulärer Kontrollfunktionen, die in der Normalzelle die Expression viraler Gene unterdrücken [11].

In Zervixkarzinomzellen sind die viralen Onkogene E6 und E7 grundsätzlich aktiv. Sie sind jeweils eigenständige Onkogene, ergänzen sich jedoch in der Transformation von Zellen. Das E6 Protein besitzt drei Funktionen, die für die Proliferation der Zellen bedeutsam sind: es bewirkt auf indirektem Wege die Bindung von Ubiquitin an den besonders wichtigen Regulator der Zellproliferation p53 und bewirkt dessen Degradation [12]. Die Folge ist ungebremstes Zellwachstum auch im Fall von auftretenden DNA-Schäden. Auf dieser Basis wirkt E6 als Mutagen. Als weiterer wichtiger Effekt bindet E6 am Carboxy-terminalen Ende die so genannten PDZ (PSD-95, Dlg, and ZO-1) Proteine und bewirkt deren funktionelle Hemmung [13-15]. Da diese Proteine eine wichtige Rolle in der Signaltransduktion spielen, wird durch deren Blockade nachhaltig in das Zellgeschehen eingegriffen. Schließlich stimuliert das E6 Protein über einen komplexen Mechanismus das Enzym Telomerase, dessen Verlust eine wichtige Rolle in der Zellalterung spielt.

Das E7 Protein greift in andere Signalwege der Zelle ein: Es bindet das Tumorsuppressorprotein $\mathrm{pRb}$ und führt zu dessen Degradation. pRB bindet in hypophosphoryliertem Zustand Mitglieder der Transkriptionsfaktor-Familie E2F, die durch die E7-pRb Bindung freigesetzt werden und Replikationsvorgänge auslösen können [16]. Darüber hinaus stimuliert es direkt die Zyklin E und A Aktivität, die für den Übergang der Zellen aus dem Ruhezustand in G1 in die S-Phase sorgen [17,18]. Schließlich bewirkt das E7 Protein Teilungsstörungen der Zentrosomen und bewirkt auf dieser Basis die Entwicklung von Aneuploidie [19].

In proliferationsfähigen Zellen der Basalmembran sind beide Onkoproteine durch zelluläre Genfunktionen weitgehend unterdrückt - erst im Verlauf der Differenzierung in suprabasalen Schichten werden diese Suppression aufgehoben und damit virale Genexpression und auch Virusproduktion ermöglicht. Die Karzinogenese durch diese Viren ist ein „Unfall“, der durch Aufhebung zellulärer Kontrollfunktionen, die intrazellulär und interzellular (parakrin) aktiv sind, bedingt wird. Hinzu kommt noch das Nicht-Erkennen der veränderten Zellen durch das Immunsystem, in der Regel bedingt durch mutative Veränderungen in Antigen-präsentierenden Signalketten. Schematisch sind diese Vorgänge in Abb. 2 wiedergegeben.

Die Übergangsphase von der Primärinfektion bis zur Krebsentstehung dauert in der Regel 20 bis 25 Jahre. Vermutlich reflektiert dies die Notwendigkeit des Auftretens spezifischer Mutationen, bedingt durch E6 und E7 im Wirtszellgenom, und den zeitaufwändigen Selektionsprozess für die auswachsenden Klone. Chemische (z.B. Tabakrauchen) und physikalische (z.B. Strahlen), oder auch andere biologische Mutagene (Infektionen mit mutagenen Eigenschaften) können den Zeitablauf deutlich beschleunigen. Im Gegensatz aber zur vielfältig auch in der Fachliteratur geäußerten Meinung gibt es aufgrund der Mutagenität der E6 und E7 Proteine gute Hinweise dafür, dass die Hr-HPV-Infektion für sich selber ausreichend sein kann, um malignes Wachstum auszulösen [5].

Verfahren des Nukleinsäurenachweises haben die Frühdiagnostik persistierender Hr-HPV-Infektionen entscheidend erleichtert und damit die Identifizierung von Risikopatientinnen für Zervixkarzinome beeinflusst. An dieser Stelle kann auf neue Nachweisverfahren nicht eingegangen werden. Es soll aber betont werden, dass neben dem direkten Virusnachweis auch indirekte Verfahren, z.B. die relativ spezifische Induktion eines zellulären Proteins $\left(\mathrm{p} 16^{\mathrm{INK} 4}\right)$ in $\mathrm{Hr}-\mathrm{HPV}$-haltigen, veränderten Zellen einen empfindlichen Nachweis erlauben. Die Serologie spielt bisher keine Rolle in der Frühdiagnostik, da serologische Reaktionen oft erst in der Spätphase bei vorhandenen Läsionen auftreten.

Spezifische therapeutische Eingriffe, welche die Virusinfektion beseitigen, stehen zurzeit nicht zur Verfügung, obwohl weltweit an solchen Möglichkeiten gearbeitet wird. Vor allem soll die Ent- 


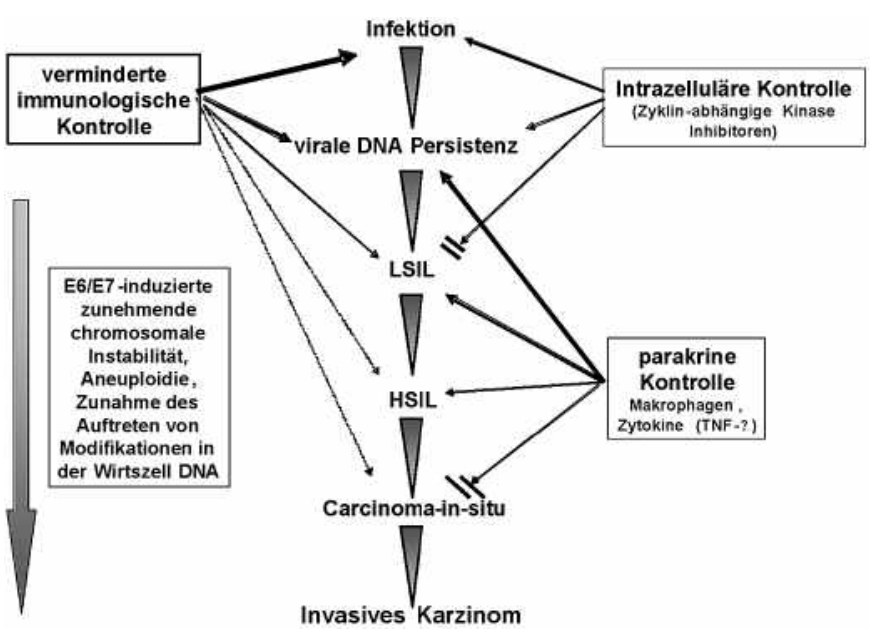

Abb. 2 Schematische Dartsellung der Pathogenese des Zervixkarzinoms. LSIL steht für "low grade squamous intraepithelial lesion“, HSIL für "high grade squamous intraepithelial lesion".

wicklung therapeutischer Vakzinen weiterhelfen, wiewohl bisher vorliegende Ergebnisse wenig befriedigend ausgefallen sind. Die topische Behandlung kutaner Läsionen mit Imiquimod scheint über Immunmodulation deutliche Erfolge zu versprechen.

\section{Papillomvirus-Infektionen der Haut}

Das vermehrte Auftreten von Warzen unter Immunsuppression bei Allograft-Transplantationen von Organen und bei immunsupprimierenden Infektionen (HIV) gilt als deutlicher Hinweis für die regulierende Rolle des Immunsystems für PapillomvirusInfektionen der Haut. Mit zeitlicher Verzögerung - aber letztlich in gewisser Parallelität zum Warzenauftreten kommt es gerade bei Transplantatpatienten auch zum erheblich vermehrten Auftreten von Plattenepithelkarzinomen der Haut. Bereits Ende der 70er-Jahre des vergangenen Jahrhunderts waren von Orth in Paris und Jablonska in Warschau spezifische Papillomvirus-Typen (vor allem HPV 5) in Plattenepithelkarzinomen von Epidermodysplasia verrucifortmis Patienten mit einer gewissen Regelmäßigkeit nachgewiesen worden [19]. Bei dieser seltenen hereditären Erkrankung kommt es vor allem an sonnen-exponierten Hautbereichen zum vermehrten Auftreten papillomatöser Veränderungen, die in einem Zeitrahmen von annähernd 20-25 Jahren in Karzinome übergehen können. Über die Analyse von Virusisolaten dieser Patientengruppe wurde eine größere Untergruppe (Genus $\beta$ ) von Epidermodysplasia verruciformis (EV)-assoziierten Viren identifiziert [4]. Frühzeitig kam es zu Spekulationen, dass auch bei nicht-immunsupprimierten Patienten auftretende Plattenepithelkarzinome der Haut etwas mit Papillomvirus-Infektionen zu tun haben könnten.

Eingehende Untersuchungen der letzten Jahre haben belegt, dass Plattenepithelkarzinome der Haut in der Mehrzahl der untersuchten Biopsien in der Tat Papillomvirus-Infektionen aufweisen. Der Prozentsatz positiver Fälle liegt bei empfindlichen Untersuchungsverfahren über 50\% [20-23]. Auch die normale Haut ist häufig HPV-positiv, allerdings in geringerem Umfang.

\section{UV-Bestrahlung der Haut}

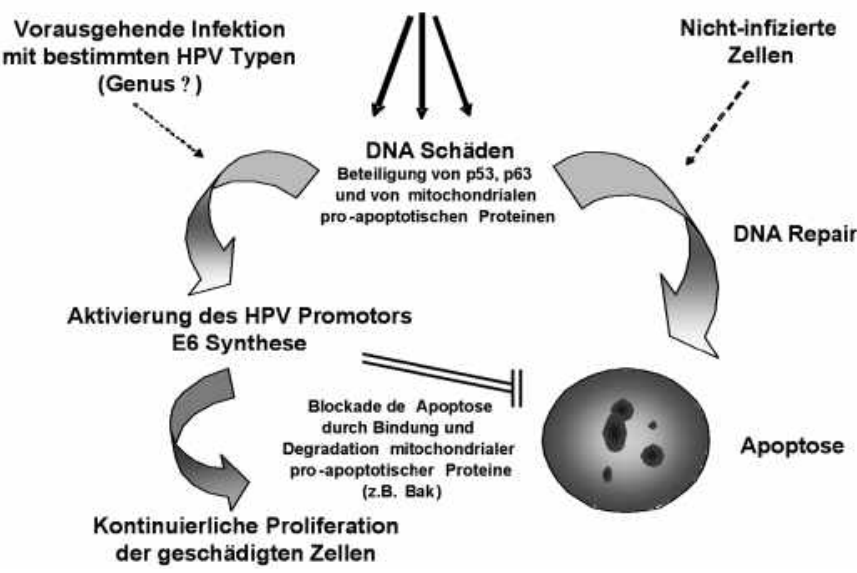

Abb. 3 Schematische Wiedergabe der Vorstellungen zur indirekten Beteiligung kutaner Papillomvirus-Typen an der Entstehung von Plattenepithelkarzinomen der Haut.

Die HPV-Infektion der Plattenepithelkarzinome der Haut unterscheidet sich deutlich von den Hr-HPV-Infektionen etwa der Zervixkarzinome: es gibt keinen analog vorherrschenden Virustyp wie HPV 16 beim letzt genannten Tumor. Vielmehr lässt sich hier ein breites Spektrum unterschiedlicher Typen des Genus $\beta$ aufzeigen, mit einer leichten Häufung der Typen 20, 23, 38 und einiger noch nicht völlig charakterisierter HPV-Genotypen. Ein weiterer Unterschied besteht in der Kopienzahl viraler Genome in den Tumorzellen: während beim Zervixkarzinom alle Krebszellen zumeist multiple Kopien viraler Genome aufweisen, sind beim Hautkrebs regelmäßig weniger als eine Genomkopie pro Zelle vorhanden. Das bedeutet, dass nur ein kleinerer Anteil der entsprechenden Tumorzellen noch Virus-positiv ist. Diese Ergebnisse legten von vornherein nahe, dass eine mögliche Beteiligung dieser Viren an der Entstehung solcher Tumoren auf einem anderen Mechanismus beruhen muss, als bei anogenitalen Infektionen mit Hr-HPVs.

In den letzten Jahren mehren sich die Hinweise dafür, dass es eine indirekte Beteiligung von Papillomvirus-Infektionen an der Entstehung der Plattenepithelkarzinome der Haut gibt. Experimentelle Untersuchungen haben gezeigt, dass spezifische Virustypen, etwa HPV 20 und HPV 38, in einem komplexen Zusammenspiel mit UV-reaktiven Zell- und Differenzierungsregulatoren, p53 und p63, aktiviert werden und frühe Proteine exprimieren [24-26]. Das E6 Protein solcher Viren (auch gezeigt für HPV 77) ist in der Lage, pro-apoptotische zelluläre Proteine zu binden und funktionell zu inaktivieren. Auf dieser Basis wird in Zellen, die durch die Sonnenlicht-bedingte UV-Einstrahlung geschädigt wurden, die Apoptose verhindert und das weitere Wachstum solcher mutierter Zellen begünstigt. Beim Auswachsen veränderter Klone sind vermutlich virale Genfunktionen nicht länger benötigt. Insgesamt gesehen unterliegen im Augenblick diese Befunde noch einer spekulativen Interpretation und bedürfen weiterer experimenteller und epidemiologischer Kontrolluntersuchungen. Abb. 3 gibt die bisher vorliegenden Vorstellungen für eine indirekte Beteiligung von HPV Infektionen bei Plattenepithelkarzinomen wieder. 
Für Basalzell-Karzinome ergeben sich derzeit noch keine Befunde, die für eine Beteiligung von Papillomvirus-Infektionen an ihrer Entstehung sprechen.

\section{Kontrolle der Papillomvirus-Infektionen}

Im Hinblick auf die Bedeutung anogenitaler Hr-HPV Infektionen für die Krebsentstehung bei Frauen wurden innerhalb des vergangenen Jahrzehnts Impfstoffe entwickelt, die vermutlich im Jahre 2006 zur Verfügung stehen werden. Sie basieren auf der Eigenschaft der viralen Strukturproteine (L1 und L2), sich nach biotechnologischer Herstellung in Hefe- oder Insektenzellen spontan zu Partikel-ähnlichen Strukturen zusammen zu lagern, die als VLP („virus-like particles“) DNA-frei isoliert und gereinigt werden können. Bisherige klinische Teste solcher Impfstoffe habe sich als überraschend effektiv erwiesen - nicht nur im Hinblick auf die Induktion humoraler und Zell-vermittelter Reaktivität, sondern auch in der Verhinderung des Auftretens prä-maligner Veränderungen (CIN III) [27-28]. Die bisher vorliegenden Impfstoffe richten sich gegen die Typen 16,18 , sowie gegen 6 und 11. Sie sollten etwa 70\% der auftretenden Zervixkarzinome und $90 \%$ aller genitalen Warzen verhindern können. Der Impfschutz scheint über mindestens 3 Jahre anzuhalten. Grundsätzlich sollte die Entwicklung polyvalenter Impfstoffe unter Einschluss weiterer Typen möglich sein. Allerdings zeichnet sich ab, dass vor allem gegen L2 gerichtete Antikörper offensichtlich gruppen-spezifische antigene Domänen erkennen können und somit den Impfschutz auch ohne Einschluss weiterer Typen möglicherweise deutlich verbreitern.

Sollten sich diese Impfungen auch weiterhin so erfolgreich erweisen, wie in den ersten klinischen Testen berichtet, so könnten theoretisch bei globaler Impfung von Jugendlichen vor Einsetzen der sexuellen Aktivität zwischen 12 und 15\% aller bei Frauen auftretenden Krebserkrankungen vermieden werden. Unter Zugrundelegung der gegenwärtigen globalen Krebsinzidenz von etwa 10 Millionen Neuerkrankungen pro Jahr wären dies zwischen 600000 bis 750000 Krebsfälle. Da solche Zahlen jedoch globale Impfprogramme erfordern, werden sich diese Ergebnisse kaum in absehbarer Zeit realisieren lassen.

\section{Ausblick}

Die Forschung über humane Papillomviren hat vor gut 30 Jahren ihre Intensivierung erfahren und in den vergangenen 23 Jahren einen enormen Aufschwung erlebt. Sie hat zur Aufklärung der infektiösen Ätiologie eines beträchtlichen Anteils menschlicher Krebserkrankungen geführt. Als praktische Konsequenz ergeben sich hieraus neue diagnostische Nachweisverfahren, vermutlich aber noch bedeutsamer wirksame Kontrollmöglichkeiten für die weltweit zweit- oder dritthäufigste Krebsform bei Frauen durch Impfungen. Diese Forschung darf als ein exzellentes Beispiel für die heute so vielfältig angesprochene translationale Forschung gelten. Sie zeigt aber auch, dass intensive Grundlagenforschung Vorraussetzung für die klinische Anwendung ist.

\section{Literatur}

${ }^{1}$ zur Hausen $\mathrm{H}$. Roots and perspectives of contemporary papillomavirus research. J Cancer Res Clin Oncol 1996; 122: 3-13

2 Dürst M, Gissmann L, Ikenberg H, zur Hausen H. A papillomavirus DNA from a cervical carcinoma and its prevalence in cancer biopsy samples from different geographic regions. Proc Nat Acad Sci US. 1983; 80: $3812-3815$

${ }^{3}$ Boshart M, Gissmann L, Ikenberg H, Kleinheinz A, Scheurlen W, zur Hausen $\mathrm{H}$. A new type of papillomavirus DNA, its presence in genital cancer and in cell lines derived from genital cancer. EMBO J. 1984; 3 : $1151-1157$

${ }^{4}$ de Villiers E-M, Fauquet C, Broker TR, Bernard H-U, zur Hausen H. Classification of papillomaviruses. Virology 2004; 324: 17-27

${ }^{5}$ zurHausen H. Papillomaviruses and cancer: from basic studies to clinical application. Nature Rev Cancer 2002; 2: 342 - 350

${ }^{6}$ Schwarz E, Freese UK, Gissmann L, Mayer W, Roggenbuck B, zur Hausen $\mathrm{H}$. Structure and transcription of human papillomavirus type 18 and 16 sequences in cervical carcinoma cells. Nature 1985; 314: 111 114

${ }^{7}$ McDougall JK. Immortalization and transformation of human cells by human papillomavirus. In: zur Hausen $\mathrm{H}$ (ed). Human Pathogenic Papillomaviruses. Berlin: Springer, 1994: $101-119$

8 IARC. Handbook on Cancer Prevention. IARC Press, 2005

${ }^{9}$ Guiliano AR, Harris R, Sedjo RL, Baldwin S, Roe D, Papenfuss MR, Abrahamsen $\mathrm{M}$, Inserra $\mathrm{P}$, Olvera $\mathrm{S}$, Hatch $\mathrm{K}$. Incidecne, prevalence, and clearance of type-specific human papillomavirus infections: The Young Women's Health Study. J Infect Dis 2002; 186: 462 - 469

${ }^{10}$ Muñoz N, Mendez F, Posso H, Molano M, van den Brule AJ, Ronderos M, Meijer CMJ, Muñoz A. Incidence, duration and determinants of cervical human papillomavirus infection in a cohort of Colombian women with normal cytology results. J Infect Dis 2004; 190: 2077-2087

${ }^{11}$ zur Hausen H. Papillomavirus infections - a major cause of human cancers. Biochem Biophys Acta Rev on Cancer 1996; 1288: F55 - F78

12 Huibregste JM, Scheffner MM, Howley PM. A cellular protein mediates association of p53 with the E6 oncoprotein of human papillomavirus typed 16 or 18. EMBO J 1991; 10: 4129-4135

${ }^{13}$ Lee SS, Weiss RS, Javier ET. Binding of human virus oncoproteins to $\mathrm{hDlg} / \mathrm{SAP97}$, a mammalian homolog of the Drosophila discs large tumor suppressor protein. Proc Natl Acad Sci USA 1997; 94: 6670-6675

${ }^{14}$ Lee SS, Glaunsinger B, Mantovani F, Banks L, Javier RT. Multi-PDZ domain protein MUPP1 is a cellular target for both adenovirus E4-ORF1 and high risk papillomavirus type 18 E6 proteins. J Virol 2000; 74: 9680-9693

${ }^{15}$ Nguyen ML, Nguyen MM, Lee D, Griep AE, Lambert PF. The PDZ ligand domain of the human papillomavirus type $16 \mathrm{E} 6$ protein is required for E6's induction of epithelial hyperplasia in vivo. J Virol 2003; 77: 6957-6964

${ }^{16}$ Dyson N, Howley PM, Münger K, Harlow E. The human papilloma virus-16 E7 oncoprotein is able to bind to the retinoblastoma gene product. Science 1989; 243: 934-937

${ }^{17}$ Dyson N, Guida P, Münger K, Harlow E. Homologous sequences in adenovirus E1A and human papillomavirus E7 proteins mediate interaction with the same set of cellular proteins. J Virol 1992; 66: 6893-6902

${ }^{18}$ Arroyo M, Bagchi S, Raychaudhuri P. Association of the human papillomavirus type 16 E7 protein with the S-phase-specific E2F-cyclin A complex. Mol Cell Biol 1993; 13: 6537-6546

${ }^{19}$ Duensing S, Münger K. Mechanisms of genomic instability in human cancer: insights from studies with human papillomavirus oncoproteins. Int J Cancer 2004; 109: 157-162

${ }^{20}$ Orth G. Epidermodysplasia verruciformis:a model for understanding the oncogenicity of human papillomnaviruses. CIBA Found Symp 1986; 120: $157-174$

${ }^{21}$ Shamanin V, Glover M, Rausch C, Proby C, Leigh IM, zur Hausen H, de Villiers E-M. Specific types of HPV found in benign proliferations and in carcinomas of the skin in immunosuppressed patients. Cancer Res 1994; 54: 4610-4613

22 Shamanin V, zur Hausen H, Lavergne D, Proby C, Leigh IM, Neumann C, Hamm H, Goos M, Haustein U-F, Jung EG, Plewig G, Wolff $H$, de Villiers EM. Human papillomavirus infections in non-melanoma skin cancers from renal transplant recipients and nonimmunosuppressed patients. J Natl Cancer Inst 1996; 88: 802 - 811

23 de Jong-Tieben LM, Berkhout RJM, Smits HL, Bouwes-Bavinck JN, Vermeer BJ, van der Woude FJ, ter Schegget J. High frequency of detection 
of epidermodysplasia verruciformis-associated human papillomavirus DNA in biopsies from malignant and premalignant skin lesions from renal transplant recipients. J Invest Dermatol 1995; 105: 367 371

${ }^{24}$ Boxman ILA, Berkhout RJM, Mulder LHC, Wolkers MC, Bouwes Bavinck JN, Vermeer BJ, ter Schegget J. Detection of human papillomavirus DNA in plucked hairs from renal transplant recipients and healthy volunteers. J Invest Dermatol 1997; 108: 712 - 715

${ }^{25}$ Storey A. Papillomaviruses: death-defying acts in skin cancer. Trends Mol Med 2002; 8: 417-421

${ }^{26}$ Giampieri S, Garcia-Escudero R, Green J, Storey A. Human papillomavirus type 77 E6 protein selectively inhibits p53-dependent transcription of proapoptotic genes following UV-B irradiation. Oncogene 2004; 23: $5864-5870$

${ }^{27}$ Fei J-W, Wei Q-X, Angel P, de Villiers E-M. Differential enhancement of a cutaneous HPV promoter by $\triangle \mathrm{Np} 63 \alpha$, jun, and mutant p53. Cell $\mathrm{Cy}$ cle (im Druck, 2005)

${ }^{28}$ Koutsky LA, Ault KA, Wheeler CM, Brown DR, Barr E, Alvarez FB, Chiacchierine LM, Jansen KU and investigators P.O.P.S.. A controlled trial of a human papillomavirus type 16 vaccine. New Engl J Med 2002; 347: $1645-1651$

${ }^{29}$ Harper DM, Franco EL, Wheeler C et al. Efficacy of a bivalent L1 viruslike particle vaccine in prevention of infection with human papillomavirus types 16 and 18 in young women: a randomised controlled trial. Lancet 2004; 364: 1757-765

${ }^{30}$ Kiyono T, Foster SA, Koop JI, McDougall JK, Galloway DA, Klingelhutz AJ. Both $\mathrm{Rb} / \mathrm{p} 16 \mathrm{INK} 4 \mathrm{a}$ inactivation and telomerase activity are required to immortalize human epithelial cells. Nature 1998; 396: 84-88

\section{Buchbesprechung}

\section{Zeckenborreliose Lyme-Krankheit bei Mensch und Tier}

H. Horst (Hrsg.)

Balingen: Spitta, 2003. 265 S. mit zahlreichen meist farbigen

Abb. Kart. 39,80€. ISBN 3-934211-49-6

Das Buch „Zeckenborreliose-Lyme-Krankheit bei Mensch und Tier" ist in der 4. Auflage erschienen. Es wurde von Horst und 8 Koautoren überarbeitet und erweitert und bietet eine schöne Praxis orientierte Übersicht über die Borrelia burgdorferi Infektion, die seit 1986 nach einer internationalen Konsensuskonferenz als „Lyme-Borreliose“ bezeichnet wird. Die Entdeckungsgeschichte wird spannend dargestellt , angereichert mit einem Erlebnisbericht von Polly Murray, einer der Mütter von Lyme, die als erste auf die kindlichen Arthritiden nach Zeckenstichen aufmerksam wurden. Sehr lesenswert ist das Kapitel über die Biologie und Ökologie der Zecken von Liebisch und Liebisch . Es folgen die klinischen Beschreibungen der einzelnen Organmanifestationen.

Das labordiagnostische Kapitel ist sehr allgemein gehalten und geht nicht besonders detailliert auf die aktuellen diagnostischen Entwicklungen und Probleme bei der Interpretation der serologischen Befunde ein. Hier findet man die sehr eigenwillige Empfehlung für Laborärzte, Liquores und Gelenkpunktate generell auch ohne Anforderung des einsendenden Arztes auf BorrelienAntikörper zu untersuchen, um damit „Aufklärungsarbeit“ zu leisten. Im Kapitel Hautborreliose wird die Labordiagnostik genauer abgehandelt, allerdings ohne Hinweis auf die derzeit gültigen MIQ Richtlinien.

Die letzten Kapitel befassen sich mit andern Zeckensticherkrankungen wie Babesiose, Ehrlichiose, FSME, Zeckenborreliose bei Haus- und Nutztieren. Auf die Problematik des Zeckenbekämpfung und die Fragen der Borreliose als Berufskrankheit wird ebenfalls in eigenen Kapiteln eingegangen

Wie vom Herausgeber und Hauptautor im Vorwort erwähnt ist das Buch als Informationsquelle für die ärztliche Praxis aller Fachrichtungen, für Studenten und Patienten gedacht. Zu jedem Kapitel gibt es Literaturangaben, die teilweise auch bis 2002 aktualisiert wurden.

H. Hofmann, München 